was admitted for successful reduction of temporomandibular joint dislocation under

4 general anaesthesia. Even under general anaesthesia it was a difficult reduction as there was no positive initial reduction, so table screening was used to ensure the condyles had been repositioned (Fig. 3). Intermaxillary fixation screws with wires were also placed to prevent wide mouth opening and prevent further dislocation, which were removed three weeks later under local anaesthesia.

In this case, diagnosis was delayed which increased the difficulty of relocation. Dislocations generally tend to present with severe pain and an inability to close the mouth, however in this case, the patient could close to a certain extent and had good lip seal, with only mild discomfort. This varied presentation delayed diagnosis at his dentist in this case. The patient may have been able to avoid increased risk of morbidity associated with general anaesthesia and late relocation if diagnosis could have been made earlier.

Complex dental treatments require the patient to open wide for a prolonged amount of time, including implant surgery, and this is commonly when non-traumatic dislocations can occur. ${ }^{1}$ This letter aims to encourage dental and implant practitioners to be aware dislocation may present varyingly especially in patients with edentulous mandibles and to encourage referral acutely to the maxillofacial department if they feel unable to relocate immediately.

I. Anjari, N. S. Narayan, S. T. Crank, by email

1. Mangi Q, Ridgway P F, Ibrahim Z, Evoy D. Dislocation of the mandible. Surg Endosc 2004; 18: 554-556.

DOI: $10.1038 / 2018.179$

\section{Swallowed partial denture}

Sir, we wish to share a case of a 63-year-old who accidentally swallowed their partial denture, which replaced the upper left two premolars, whilst taking their morning medications. Despite no initial breathing difficulties or significant pain, the patient attended A\&E where they were initially seen by the local oral \& maxillofacial surgical
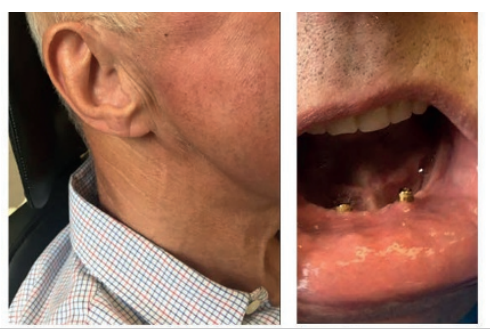

Fig. 1 Clinical representation of patient with bilateral diffuse swellings over TMJ area and partial closure of the mouth

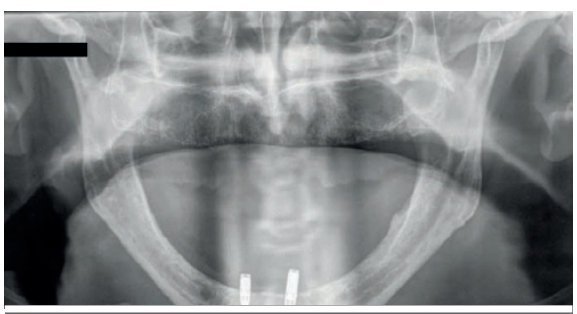

Fig. 2 An orthopantogram showing position of two mandibular dental implants and confirming bilateral dislocation of the temporomandibular joints

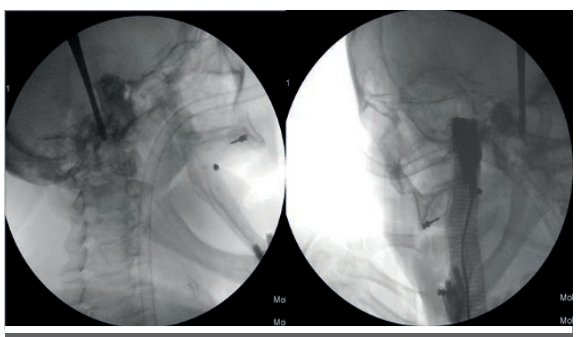

Fig. 3 Table screening whilst patient under general anaesthesia to ensure the condyles had been repositioned successfully team. CT abdomen and pelvis demonstrated 'Dentures lodged in the distal oesophagus' warranting 'urgent upper GI surgical or gastroenterological opinion'. Emergency oesophagogastroduodenoscopy by the gastroenterologists failed to identify the denture. After consultation with the general surgeons, an abdominal radiograph demonstrated the dentures' likely position having moved to the small intestines. The patient was managed non-surgically being kept in hospital for monitoring before being discharged home to await natural passage of their denture. The cost of this admission was approximately $£ 1,500$.

Reflecting upon this case highlights a number of things. A number of reports have documented such problems with small unilateral RPDs. ${ }^{1-3}$ In this case an uneventful passage of the denture occurred but more serious complications such as bowel perforation have been documented. Management of such problems usually requires involvement of the MDT within hospital. In such a setting the anatomical location of the prosthesis can be identified and appropriate arrangements can be made to remove the prosthesis either actively or passively depending on its whereabouts.

M. Lawrence, K. Fan, by email

1. Nimmo S S, Nimmo A, Chin G A. Ingestion of a unilateral removable partial denture causing serious complications.Oral Surg Oral Med Oral Pathol 1988; 66: 24-26.

2. Rizzatti-Barbosa C M, Cunha F L, Bianchini W A, de Albergaria-Barbosa J R, Gomes B P. Accidental impaction of a unilateral removable partial denture: a clinical report. J Prosthet Dent 1999; 82: 270-271.

3. Gallas M, Blanco M, Martinez-Ares D, Rivo E, GarcíaFontán E, Cañizares M. Unnoticed swallowing of a unilateral removable partial denture._Gerodontology 2012; 29: e1198-200.

DOI: $10.1038 /$ sj.bdj.2018.180
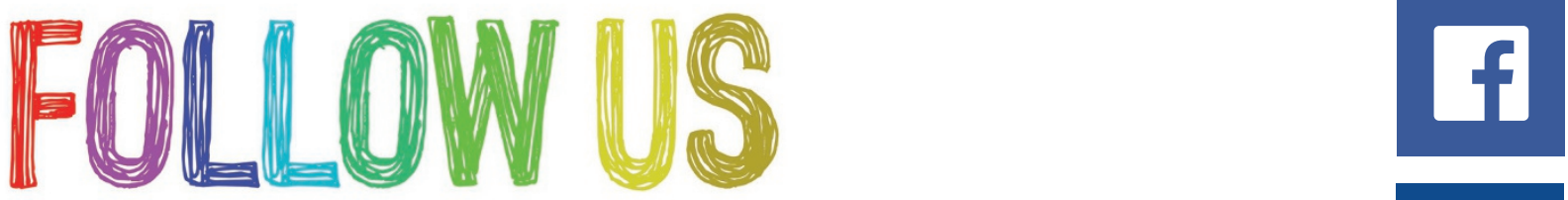

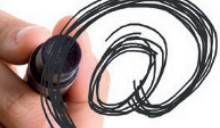 britishdentaljournal
} BDJ

\author{
facebook.com/britishdentaljournal
}

